\title{
Tardive Dyskinesia Exacerbated after Ingestion of Phenylalanine by Schizophrenic Patients
}

\author{
Diane M. Mosnik, M.S., Bonnie Spring, Ph.D., Keith Rogers, M.D., Ph.D., and Sankar Baruah, M.S.
}

\footnotetext{
Despite continued research, the influences that promote or exacerbate tardive dyskinesia (TD) symptoms remain incompletely understood. Recent findings (Gardos et al. 1992; Richardson et al. 1989) suggest that ingestion of the dietary constituent, phenylalanine, might exacerbate TD symptoms, but a double-blind, placebo-controlled challenge had not previously been conducted in schizophrenic patients. On two different mornings, in counterbalanced order, 18 male schizophrenic patients with TD were challenged with either $100 \mathrm{mg} / \mathrm{kg}$ phenylalanine or placebo. Effects on abnormal involuntary movements, recall memory, and plasma phenylalanine were measured 90 minutes post-challenge. The results supported the
}

hypothesis in that involuntary movements increased to a statistically and clinically meaningful degree after the phenylalanine challenge, but not after placebo. No effects on memory were detected. Significant order effects characterized the plasma findings but not the behavioral data. Results indicate that a dietary constituent, the amino acid phenylalanine, can potentially exacerbate tardive dyskinesia symptoms in schizophrenic patients. The influence of phenylalanine and other ingested substances on clinical symptomatology warrants further investigation. (C) 1997 American College of Neuropsychopharmacology [Neuropsychopharmacology 16:136-146, 1997]
KEY WORDS: Tardive dyskinesia; Schizophrenia; Phenylalanine; Amino acids

The extrapyramidal motor disturbance, tardive dyskinesia (TD), is associated with spontaneous involuntary movements involving bucco-oro-lingual dyskinesias and choreoathetoid movements of the trunk and limbs. Because the syndrome is unsightly and often irreversible, TD remains a serious concern in the treatment of patients who chronically receive antipsychotic medications. In addition to motor symptoms, some evidence indicates that concomitant cognitive disturbances, espe-

From the Department of Psychiatry (DMM, SB), University of Iowa College of Medicine, Iowa City, Iowa; Department of Psychology (DMM, BS), Finch University of Health Sciences/Chicago Medical School, Chicago, Illinois; Biological Psychiatry (BS), Hines Veterans Affairs Medical Center, North Hines Illinois; Psychiatry Service (KR), Seattle Veterans Affairs Medical Center, Seattle, Washington.

Address correspondence to: Diane M. Mosnik, The University of Iowa Hospitals and Clinics, MHCRC Rm 2911JPP, Department of Psychiatry, 200 Hawkins Drive, Iowa City, IA 52242.

Received September 11, 1995; revised January 24, 1996; accepted January 26, 1996. cially memory impairments, are also associated with TD (Sorokin et al. 1988; Myslobodsky et al. 1985; Wade et al. 1987).

There have been widely varying appraisals of the prevalence of TD among psychiatric populations treated with neuroleptic medications. Although estimates of TD's prevalence vary from $0.5 \%$ to $56 \%$, a prevalence rate of $20 \%$ is commonly reported (Kane and Smith 1982; Benson and Blumer 1982; Yassa et al. 1990). Among some sectors of the population, though, TD occurs with considerable higher frequency, characterizing as many as $50 \%$ of the elderly, for example (Barnes 1987).

An etiological role for the neuroleptics has certainly been demonstrated in TD (Jeste and Wyatt 1981; Owens et al. 1982). There remains, however, a marked lack of consistent evidence that systematic variation in the severity or degree of risk of developing TD can be completely explained on the basis of longer exposure to neuroleptics or exposure to higher potency neuroleptics (Kane et al. 1985; Casey 1987). In fact, age is the only variable that consistently has been shown to increment in paral- 
lel with increased vulnerability and severity of TD (Smith and Baldessarini 1980; Kane and Smith 1982; Saltz et al. 1989). Other attributes, such as female gender, length of hospitalization, or duration of neuroleptic use have not been as consistently associated (Richardson et al. 1986). Furthermore, there has been a genuine dearth of research on state-related influences that exacerbate the presentation of TD symptoms (Richardson and Casey 1988).

Some findings suggest that ingestion and metabolism of the amino acid, phenylalanine, could influence the development and exacerbation of TD (cf. Krause et al. 1985). In a study examining risk factors for TD development among mentally retarded patients who had been treated with neuroleptics, Richardson et al. (1986) found a TD prevalence of $86 \%$ among patients with phenylketonuria (PKU), compared with only $27 \%$ in the remaining sample. Because PKU is a disease characterized by elevated brain levels of phenylalanine, these findings suggested that elevated phenylalanine levels might constitute a vulnerability factor for TD.

Subsequently, Richardson and colleagues (1989) investigated whether patients with TD showed an altered metabolic response to dietary phenylalanine. Even when fasting, subjects with TD exceeded non-TD patients in their ratio of plasma phenylalanine to other large neutral amino acids (LNAAs). Differences increased in response to an oral phenylalanine challenge, which produced a significantly greater increase in plasma phenylalanine in TD patients than controls. Moreover, phenylalanine plasma levels were significantly correlated with TD severity. These results are consistent with the premise that the metabolism of dietary phenylalanine may be altered both chronically and acutely in individuals with TD.

To examine whether the behavioral as well as the metabolic response to phenylalanine is altered among patients with TD, Gardos et al. (1992) administered a challenge dose of $100 \mathrm{mg} / \mathrm{kg}$ phenylalanine to unipolar depressed patients with or without TD. He found no differences in fasting or 2-hour post-challenge plasma phenylalanine levels between groups of depressed patients with TD, those exposed to neuroleptics but lacking TD, and those never exposed to neuroleptics. On the other hand, post-loading plasma phenylalanine levels were significantly correlated with ratings on the $\mathrm{Ab}$ normal Involuntary Movements Scale (AIMS), suggesting that individuals with more severe TD may have special difficulty metabolizing phenylalanine.

In addition to findings that associate elevated plasma phenylalanine with tardive dyskinesia, a body of research indicates that plasma phenylalanine levels are significantly elevated in patients with schizophrenia. For example, Poisner (1960) found that schizophrenics showed elevated plasma phenylalanine $(2.65 \mathrm{mg} /$ centimeter) compared with controls $(1.88 \mathrm{mg} /$ centimeter; $p<.003)$. He noted that the schizophrenic's phenylalanine levels were similar to those that characterize het- erozygotes for PKU. Similarly, in a sample of 50 patients with schizophrenia, Lippman (1958) found that 13 exhibited phenylalanine values in the borderline range of heterozygosity for PKU. Moreover, there is evidence to indicate that elevated plasma phenylalanine levels characterize schizophrenic patients before the onset of TD (Bjerkenstedt et al. 1985; Reveley et al. 1987; Potkin et al. 1983).

As both tardive dyskinesia and schizophrenia are associated with elevated plasma phenylalanine, phenylalanine may be especially increased in individuals who suffer from both syndromes. It is possible to propose at least one pathway by which a heightened plasma phenylalanine level could exacerbate tardive dyskinesia. In addition to noradrenergic (Wolf and Mosnaim 1988; Nasrallah et al. 1986) and GABAergic influences (Fibiger and Lloyd 1984), dopamine is thought to play a role in the pathophysiology of tardive dyskinesia. The dopamine-receptor supersensitivity hypothesis states that chronic blockade of dopamine-receptor sites causes a functional decrease in dopamine input to the striatum, resulting in compensatory supersensitivity of that dopamine system and, ultimately, increased dopaminergic activity, which leads to TD (Ebadi and Hama 1988). Consequently, an increase in dopamine would aggravate TD symptomatology, as demonstrated by the action of L-Dopa and amphetamine (Carroll et al. 1977).

Heightened phenylalanine can yield increased dopamine via the irreversible hydroxylation of phenylalanine to tyrosine by phenylalanine hydroxylase (Harper 1984). Tyrosine is the immediate precursor of the brain catecholamine neurotransmitters, dopamine and norepinephrine. When neuronal activity is increased, as might characterize nigrostriatal dopamine neurons in tardive dyskinesia, catecholamine synthesis becomes increasingly affected by precursor availability (Milner et al. 1986). It has been demonstrated that enhanced tyrosine availability increases catecholamine synthesis or release and augments catecholamine-dependent behaviors (Melamed et al. 1980). When tyrosine is low and phenylalanine is high, as should result from reduced tyrosine transport in patients with schizophrenia and tardive dyskinesia (Bjerkenstedt 1990), phenylalanine could support catecholamine synthesis. Low doses of phenylalanine ( $200 \mathrm{mg} / \mathrm{kg}$ in rats) have been shown to increase dopamine release by 59\% (During et al. 1988) and would therefore be expected to exacerbate TD symptoms.

Phenylalanine, an essential amino acid, cannot be synthesized by the body and must be obtained via ingestion of food. Because all of the large neutral amino acids (LNAAs) enter the brain via a common transport mechanism, phenylalanine's brain influx is affected by its ratio to all other LNAAs (tyrosine, tryptophan, valine, leucine, isoleucine, histidine, and methionine) (Pardridge and Choi 1986; Hagenfeldt et al. 1984; Fern- 
strom and Faller, 1978). Paradoxically, although the ingestion of protein supplies some phenylalanine and therefore elevates plasma phenylalanine, protein intake actually decreases brain phenylalanine influx because of the simultaneous rise in competing LNAAs (Fernstrom and Wurtman 1972).

In contrast, consumption of pure phenylalanine or aspartame, which consists of $56 \%$ phenylalanine (Stegink and Filer 1984), has the potential to increase brain phenylalanine disproportionately, partly because of the lack of competing amino acids (Maher 1990) and partly because phenylalanine's affinity for the LNAA transporter is greater than that of the other LNAAs (Pardridge and Oldendorf 1977). Ingestion of phenylalanine together with carbohydrate magnifies this effect because carbohydrate intake elicits the release of insulin, which selectively lowers plasma levels of the branched-chain amino acids while lacking much effect on phenylalanine. Consequently, if an aspartame-containing beverage is consumed along with a carbohydrate-rich, protein-poor food, brain phenylalanine influx may be almost doubled (Hernandez and Coulson 1961; Wurtman 1983).

To summarize, current evidence indicates that elevated plasma phenylalanine levels are associated with TD risk, TD severity, and schizophrenia. Because food is the principal source of phenylalanine, we tested the prediction that a dietary phenylalanine challenge would exacerbate the motor and cognitive accompaniments of TD in patients with schizophrenia. Although a prior test of a phenylalanine challenge had been undertaken by Gardos et al. (1992), that study involved patients with depression rather than schizophrenia and had not utilized a double-blind, placebo-controlled design. Our primary hypothesis was that patients with schizophrenia would display an acute increase in tardive dyskinetic symptoms after ingestion of a phenylalanine loading dose but not after ingestion of an equivalent placebo. We also hypothesized that cognitive performance on a test of memory recall would be poorer after ingestion of phenylalanine than placebo.

\section{METHODS}

\section{Sample}

Eight of the 18 patients were recruited from mental health clinics in Iowa (the University of Iowa Hospitals and Clinics, the Iowa City Veterans Affairs Hospital, and community mental health centers in Independence and Muscatine). The remaining 10 patients came from the North Chicago Veterans Affairs Hospital inpatient and outpatient services. The study protocol was reviewed and approved by the Institutional Review Boards at each recruitment site. All subjects held a current DSM-III-R hospital diagnosis of schizophrenia (American Psychiatric Association 1987). Diagnoses were verified using a modified semi-structured interview by the principal investigator and the patient's treating physician, as well as a review of medical records.

An additional section criterion was that both the first author and an independent rater were required to agree that each subject met criteria for the current presence of clinically significant TD. Significant TD was defined as an Abnormal Involuntary Movements Scale (NIMH 1975) rating of two on at least two body areas, or a score of at least three on any single body area (Schooler and Kane 1982).

All patients were male, and candidates were excluded from the study if they declined to participate, were incompetent to provide informed consent, or failed to display TD symptomatology. To minimize sample heterogeneity, patients with obesity, phenylketonuria, diabetes, significant past or current affective symptomatology, current drug or alcohol abuse, epilepsy, or systemic illness known to cause psychiatric symptoms were also excluded. Patients with idiopathic Parkinson's disease were not studied either to eliminate conditions that might impede an accurate diagnosis of tardive dyskinesia. All patients were screened specifically for signs of tardive dyskinesia and therefore were not studied if they displayed signs of dystonia or torticollis. In addition, although several patients displayed akathisia, these symptoms were not assessed. All patients displayed TD symptoms involving facial regions, i.e., buccaloral-masticatory movements of tongue, cheeks, jaw, and forehead, and somewhat less commonly movements of the fingers/toes. In addition, some patients displayed TD in arms, feet, and trunk regions.

\section{Procedures}

The study design is illustrated in Figure 1.

Patients were randomized to receive either phenylalanine first (day 1$)$ followed by placebo (day 2) $(n=10)$ or the opposite order $(n=8)$. Before each test day, subjects fasted from 10 P.M. in order to reduce potential confounding effects of other dietary intake. Upon awakening, they continued to fast and reported to the laboratory for the test sessions, which typically occurred on two consecutive days. After the patient was seated quietly in a room, AIMS ratings were completed to assess baseline TD severity for that day. Next, a 5-ml blood sample was drawn to measure baseline amino acid levels. Next, at approximately 8:00 A.M., patients ingested the challenge drink. Drinks were prepared by an assistant and administered by the first author in a doubleblind manner. Ninety minutes after the challenge drink was consumed, indicated by pilot testing to be the peak rise in plasma phenylalanine, subjects were again rated on TD severity and a second blood sample was drawn. Finally, the memory recall test was administered. During the 90-minute waiting period, subjects were ques- 
Figure 1. Overall study design. AVLT indicates Rey Auditory Verbal Learning Test.

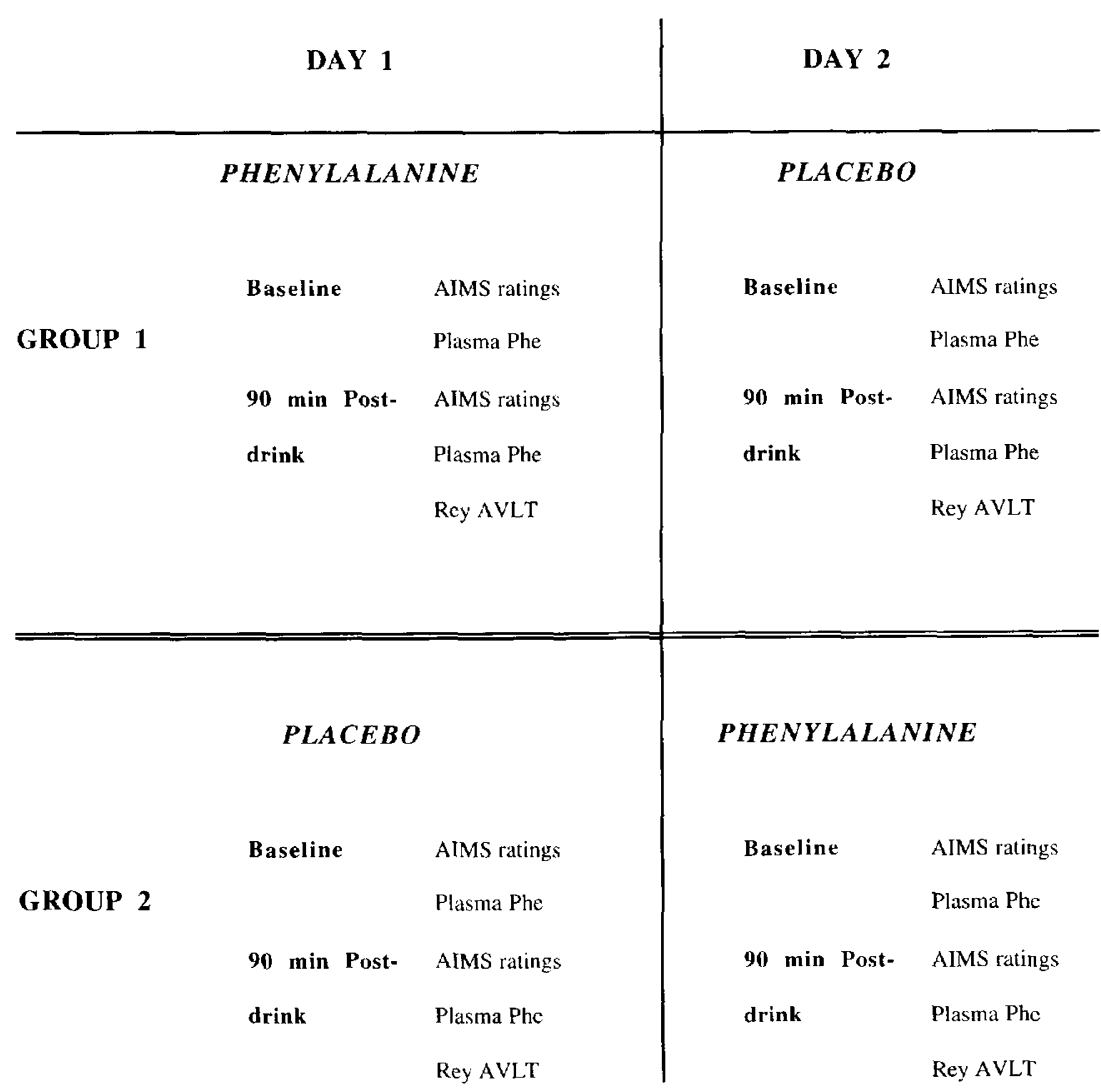

tioned about their symptoms and monitored while they rested and smoked cigarettes. Standing doses of prescribed medications were administered as usual on study days.

\section{Challenge Drinks}

The active drink consisted of powdered L-phenylalanine (100 mg/ $\mathrm{kg}$ body weight), which was mixed with three scoops of powdered orange-flavored breakfast drink (Tang) and dissolved in $500 \mathrm{ml}$ of water. L-phenylalanine was supplied by Ajinomoto USA, Inc. (Raleigh, North Carolina) under approval from the FDA. The placebo drink consisted of three scoops of the powdered breakfast drink dissolved in $500 \mathrm{ml}$ of water. The fine white L-phenylalanine powder could not be tasted or detected visually when mixed with the bright orangecolored powder. The breakfast drink was selected as the vehicle because it lacked protein and contained sucrose, an insulin-stimulating carbohydrate, which, via insulin release, would decrease branched-chain amino acids in plasma, contributing to elevation of the phenylalanine ratio.

\section{Behavioral Measures}

Dyskinetic Movements. The AIMS rating scale is a well-validated tool for assessing tardive dyskinesia (Simpson et al. 1979; Schooler and Kane 1982). The ver- sion used in this study consisted of 11 items designed to assess the occurrence of dyskinetic movements in three main body areas. Each item was rated for severity on a scale ranging from 0 (absent) to 4 (severe). Thus, a person could meet entry criteria by receiving a high score $(3+)$ on only one defined movement. Alternatively, if abnormal movements were observed in more than one region, he could qualify by receiving $2+$ scores in at least two body areas. The scale also included summary ratings for overall severity (highest single score on any body region), overall asymmetry, patient awareness of movements, and total score (sum of severity in all body regions).

Recall Memory. Parallel forms of the Rey Auditory Verbal Learning Test (AVLT) were used to assess memory recall after ingestion of each substance (Rey 1964; Taylor 1959). The AVLT entails five presentations of a 15-word list with each presentation followed by an immediate recall trial. The primary score analyzed was recall on trial 5. Equivalent forms $\mathrm{A}$ and $\mathrm{C}$ were used to minimize practice effects on the second administration of the test.

Plasma Amino Acids. Blood was drawn into vacutainer tubes containing EDTA as an anticoagulant. The tubes were then centrifuged at $750 \mathrm{~g}$ for 15 minutes, after which the plasma was aspirated into labeled tubes and stored at $-70^{\circ} \mathrm{C}$ for later analysis. At the time of 
analysis, $0.1 \mathrm{ml}$ of $1 \mathrm{mmol} / \mathrm{L}$ allothreonine (Sigma Chemical Co.) was added as an internal standard to $0.5 \mathrm{ml}$ of thawed plasma, followed by $0.4 \mathrm{ml}$ of $10 \%$ trichloroacetic acid (TCA) (Fisher Scientific). Samples were then kept on ice for 10 minutes to completely precipitate the protein and centrifuged at $16,000 \mathrm{~g}$ for 3 minutes. The supernatant was then filtered through a 0.2 micron Acrodisc filter (Gelman).

Quantitative amino acid analysis was performed at the University of Iowa Hospitals and Clinics psychiatric research laboratories. Peak areas for the large neutral amino acids, tyrosine, tryptophan, valine, leucine, isoleucine, threonine, methionine, histidine, and phenylalanine, were measured after precolumn derivatization with o-phthaldehyde (OPA) (Fluka Chemical Corp.) according to the method of Lindroth and Mopper (1979) and Lenda and Svenneby (1980), with minor modifications. Samples were diluted two-fold with $20 \mathrm{mmol} / \mathrm{L}$ phosphate buffer, $\mathrm{pH} 7.4$, before derivatization. Twenty (20) $\mu \mathrm{l}$ of sample was mixed with $60 \mu \mathrm{l}$ of $2 \mathrm{mg} / \mathrm{ml}$ OPA containing $0.01 \%$ 2-mercaptoethanol (2-ME) (Fluka). Samples were derivatized for 2 minutes before injection. Derivatized amino acids were then separated using reverse-phase high performance liquid chromatography (HPLC) over a 5-micron, C-18, 15-cm column, "Ultima" (Alltech). The column was maintained at $40^{\circ} \mathrm{C}$ using a Dupont column heater. A Gilson autosampler model 231 was routinely used for derivatization and injection. Amino acids were separated with eluent $\mathrm{A}=50$ $\mathrm{mmol} / \mathrm{L}$ sodium acetate, $\mathrm{pH} 5.6$ (Fisher Scientific), with $4 \%$ acetonitrile (EM Science); eluent B = methanol (EM Science).

The HPLC instrumentation consisted of a SCL-6A system controller, two LC-6A pumps, and an RF-530 fluorescence detector, all from Shimadzu. The detector was set at an excitation wavelength of $345 \mathrm{~nm}$ and emission wavelength of $445 \mathrm{~nm}$. Limits of detection were $0.34 \mathrm{pM}$ with a coefficient of variation of $10 \%$. Data were collected using a Shimadzu C-R4A chromatopac and analyzed by determining the ratio of phenylalanine peak area to the LNAA peak areas.

\section{Statistical Analyses}

The data were analyzed using a mixed model repeated measures analysis of variance (ANOVA) to detect changes in TD severity depending on treatment condition (phenylalanine vs. placebo). The grouping factor was order of ingestion (phenylalanine first vs. placebo first). The repeated measures factors were challenge substance (phenylalanine vs. placebo) and rating time (pre- vs. post-drink). The main hypothesis predicted a directional substance by time interaction, such that postdrink AIMS total severity was expected to increase above pre-drink values after ingestion of phenylalanine but not after ingestion of placebo. A mixed model anal- ysis of variance was also conducted to test the hypothesis that memory recall would be poorer after ingesting phenylalanine than placebo.

\section{RESULTS}

\section{Sample Characteristics}

The 18 participants ranged in age from 28 to 65 (mean = 44 years, $\mathrm{SD}=11.8$ ). Fourteen were right-handed, three were left-handed, and one was of mixed handedness (Oldfield 1971). All but four patients smoked cigarettes. All were receiving some type of antipsychotic medication at the time of testing. Demographic characteristics for the two groups defined by the order of ingestion of phenylalanine are shown in Table 1 . There were no differences between the groups on any demographic variable. One subject in the group who received phenylalanine on the first day, left the hospital against medical advice after completing only 1 day of the study; however, his data were included in the analyses.

The study procedure was well tolerated by the patients. The only complaints were of feeling tired (two subjects) and experiencing mild discomfort during blood drawing.

\section{Plasma Amino Acids}

Analysis of plasma phenylalanine levels revealed a drink $\times$ time interaction $(\mathrm{F}=178.1 ; d f=1,14 ; p=.0001)$ which was embedded in a drink $\times$ time $\times$ order interaction $(\mathrm{F}=16.7$; $d f=1,14 ; p=.001)$. As shown in Figures 2 and 3, the two-way interaction indicated that plasma phenylalanine rose significantly after the phenylalanine challenge but not after the placebo drink. The three-way interaction indicated that the magnitude of the post-challenge increase in phenylalanine differed as a function of whether patients received the phenylalanine challenge on day 1 or day 2. Patients who received phenylalanine on the second day of the study exhibited a significantly greater post-challenge rise in

Table 1. Demographic Characteristics of Groups Defined by Order of Ingestion of Phenylalanine (mean \pm SD)

\begin{tabular}{lcc}
\hline & $\begin{array}{c}\text { Phenylalanine } \\
\text { Day 1 }\end{array}$ & $\begin{array}{c}\text { Phenylalanine } \\
\text { Day 2 }\end{array}$ \\
\hline$n$ & 10 & 8 \\
Age (years) & $45.0 \pm 12.4$ & $43.0 \pm 11.1$ \\
Weight (kg) & $76.3 \pm 11.2$ & $73.7 \pm 9.1$ \\
Baseline TD Severity $_{\text {Smoking Status }}{ }^{a}$ & $12.8 \pm 3.0$ & $11.9 \pm 2.2$ \\
Handedness $^{b}$ & $0.8 \pm 0.4$ & $0.8 \pm 0.4$ \\
\hline
\end{tabular}

${ }^{a}$ Smoking status: $1=$ smoker, $0=$ nonsmoker.

${ }^{b}$ Handedness: 1 = right, $2=$ left, $3=$ mixed handedness. 


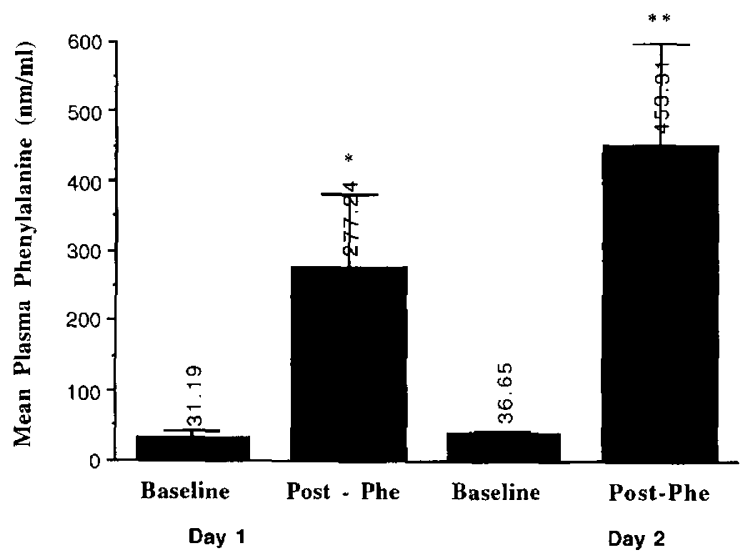

Figure 2. Mean plasma phenylalanine (in $\mathrm{nm} / \mathrm{ml}$ ) prior to (baseline) and 90 minutes post-phenylalanine challenge for subjects who ingested phenylalanine on day 1 versus those who ingested phenylalanine on day 2 . Note: both post-phenylalanine plasma levels are significantly different from baseline levels and plasma phenylalanine on day 2 is significantly different than on day 1 .

plasma phenylalanine than did patients who received phenylalanine on the first day of the study. Phenylalanine loading resulted in approximately a 12 -fold increase in plasma phenylalanine level in patients who received phenylalanine on the second day, in contrast to

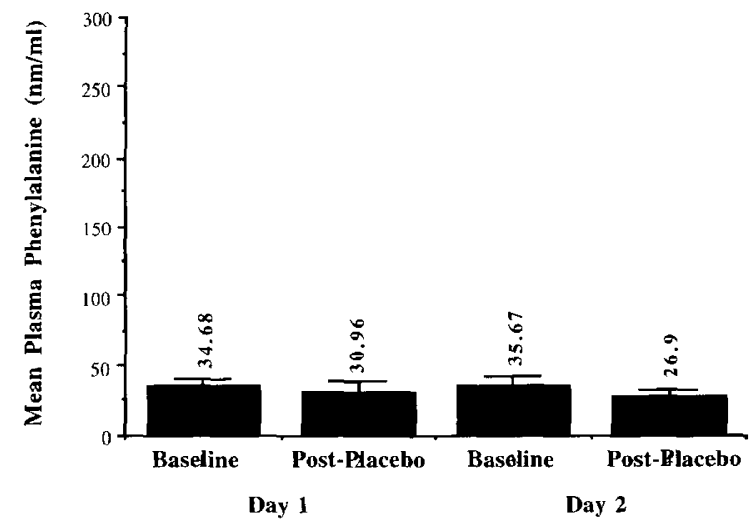

Figure 3. Mean plasma phenylalanine (in $\mathrm{nm} / \mathrm{ml}$ ) prior to (baseline) and 90 minutes post-placebo for subjects who ingested placebo on day 1 versus those who ingested placebo on day 2 .

about a nine-fold increase in patients who received phenylalanine on the first day.

Subsequent analyses of covariance controlling for differences in the two groups' weight, age, smoking status, and location (Chicago vs. Iowa) failed to identify any between-group difference that could explain the order effect. Nor could the difference in post-ingestion plasma phenylalanine on day 2 as opposed to day 1 be attributed to baseline variation as there were no signifi-

Table 2. Plasma Levels of All Amino Acids (in $\mathrm{nm} / \mathrm{ml}$ ) by Substance and by Day (mean $\pm \mathrm{SD}$ )

\begin{tabular}{|c|c|c|c|c|c|c|}
\hline \multirow[b]{2}{*}{ Amino Acids } & \multicolumn{2}{|c|}{ Baseline } & \multicolumn{2}{|c|}{$\begin{array}{c}\text { Post-ingestion } \\
\text { Day } 1\end{array}$} & \multicolumn{2}{|c|}{$\begin{array}{c}\text { Post-ingestion } \\
\text { Day } 2\end{array}$} \\
\hline & Mean & (SD) & Mean & (SD) & Mean & (SD) \\
\hline \multicolumn{7}{|l|}{ Placebo } \\
\hline Phenylalanine & 35.26 & $(5.8)$ & 30.96 & $(7.4)$ & 26.90 & (5.1) \\
\hline Tyrosine & 47.40 & $(10.2)$ & 42.97 & (11.3) & 35.17 & (6.3) \\
\hline Tryptophan & 10.56 & $(6.9)$ & 8.40 & (5.1) & 10.69 & (5.5) \\
\hline Valine & 272.33 & $(57.2)$ & 239.03 & $(32.0)$ & 226.48 & $(60.5)$ \\
\hline Leucine & 89.20 & $(18.9)$ & 71.09 & $(16.8)$ & 62.79 & (16.4) \\
\hline Isoleucine & 48.02 & $(10.0)$ & 39.37 & $(7.4)$ & 32.27 & $(8.7)$ \\
\hline Methionine & 10.77 & $(5.6)$ & 10.10 & $(4.6)$ & 8.36 & (3.6) \\
\hline Histidine & 38.22 & $(15.9)$ & 39.88 & $(16.5)$ & 33.67 & (11.1) \\
\hline Threonine & 94.48 & $(28.6)$ & 97.70 & $(29.2)$ & 75.04 & $(17.6)$ \\
\hline \multicolumn{7}{|l|}{ Phenylalanine } \\
\hline Phenylalanine & 33.74 & $(7.4)$ & 277.24 & (102.2) & 453.31 & (145.5) \\
\hline Tyrosine & 47.43 & (14.5) & 90.78 & $(18.5)$ & 91.11 & (17.5) \\
\hline Tryptophan & 11.70 & $(6.1)$ & 9.31 & $(5.1)$ & 7.09 & $(6.2)$ \\
\hline Valine & 260.00 & $(52.9)$ & 219.45 & $(45.6)$ & 231.93 & $(42.1)$ \\
\hline Leucine & 86.92 & $(21.0)$ & 51.39 & $(24.1)$ & 61.28 & (19.0) \\
\hline Isoleucine & 47.99 & $(11.5)$ & 29.43 & $(9.8)$ & 37.03 & $(12.5)$ \\
\hline Methionine & 13.14 & $(6.0)$ & 8.22 & (3.9) & 7.93 & (3.9) \\
\hline Histidine & 39.43 & $(14.6)$ & 29.53 & $(13.7)$ & 33.00 & (13.5) \\
\hline Threonine & 91.85 & $(26.6)$ & 70.87 & $(20.2)$ & 91.97 & (20.2) \\
\hline
\end{tabular}

Note: The baseline mean values for both placebo and phenylalanine are collapsed across order of ingestion. All plasma levels are given in nanomoles per milliliter. 

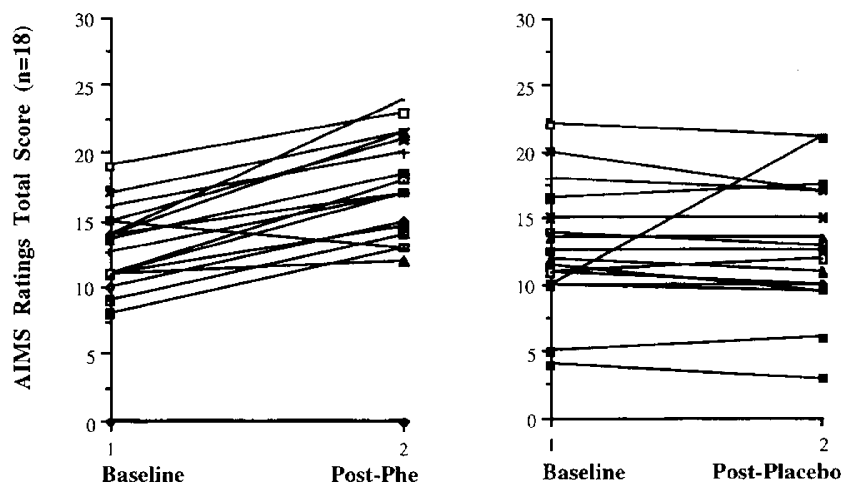

Figure 4. Left graph shows the AIMS TD severity score for each subject before and 90 minutes after ingesting phenylalanine. Right graph shows the AIMS TD severity score for each subject before and 90 minutes after ingesting placebo. Subjects from both orders of ingestion are shown together.

cant between-group differences in baseline plasma phenylalanine values on any test day (see Figure 3 ).

At post-ingestion, but not baseline, the phenylalanine ratio, [phenylalanine / (tyrosine + leucine + isoleucine + threonine + methionine + histidine + valine + tryptophan)], was also significantly greater for subjects who received the phenylalanine challenge on the second day (mean $=0.5 \pm 0.2$ ) compared with the first day $($ mean $=0.8 \pm 0.3)(p=.02)$.

Plasma tyrosine and tryptophan did not show order effects on either baseline or post-ingestational values. Plasma tyrosine rose equivalently, about two-fold, after phenylalanine loading regardless of the order of administration. Post-loading plasma tyrosine averaged $90.8 \pm$ $18.5 \mathrm{~nm} / \mathrm{ml}$ when the phenylalanine challenge was given on day 1 , and $91.1 \pm 17.5 \mathrm{~nm} / \mathrm{ml}$ when the challenge was on day 2. In contrast to the post-challenge rise in phenylalanine and tyrosine, tryptophan decreased slightly after the phenylalanine challenge. The observed changes in plasma phenylalanine, tyrosine, and tryptophan were similar in magnitude to those reported elsewhere (Gardos et al. 1992; Richardson et al. 1989). The mean plasma levels for all measured amino acids (in $\mathrm{nm} / \mathrm{ml}$ ) are displayed by time in Table 2 .

\section{AIMS Scores}

The mixed model repeated measures analysis of variance with AIMS total score as the dependent measure detected a significant drink by time interaction ( $\mathrm{F}=$ $16.77 ; d f=1,14 ; p=.001)$. Simple effects analyses to interpret the interaction indicated that AIMS scores increased significantly 90 minutes after the phenylalanine challenge but did not change after ingestion of placebo. Comparable changes in TD symptomatology after phenylalanine loading were noted for both face and body subregions. A post-phenylalanine worsening of TD symptoms occurred with striking consistency, characterizing

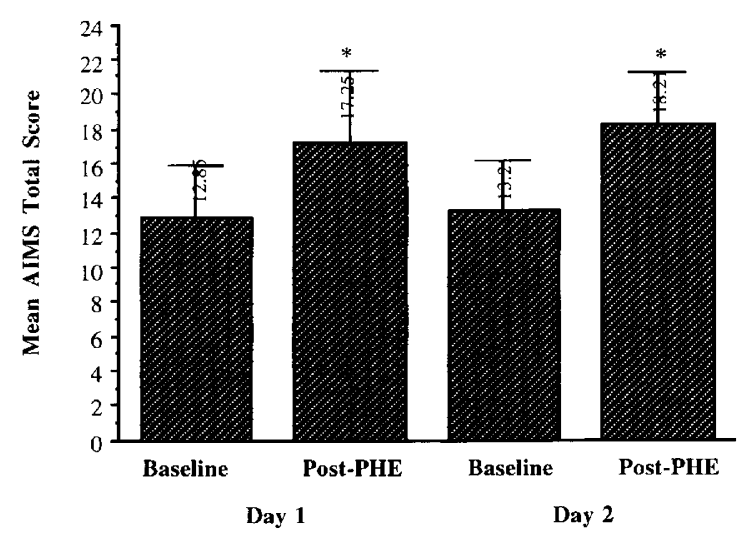

Figure 5. Mean AIMS TD severity score before and $90 \mathrm{~min}-$ utes after phenylalanine challenge for subjects who ingested phenylalanine on day 1 versus those who ingested phenylalanine on day 2. Both post-phenylalanine ratings are significantly different from baseline ratings.

all but one patient. Figure 4, showing baseline and postdrink AIMS scores for each subject, demonstrates that TD symptoms consistently remained stable after placebo and worsened after phenylalanine, even though the drinks were given and the TD ratings were performed double-blind. Moreover, the change in AIMS scores after ingestion of phenylalanine appeared to be clinically meaningful, because a majority of subjects $(n=$ 12/18) exhibited total AIMS score increases of at least 3 points, indicating significant worsening of TD severity (DeVeaugh-Geiss 1988).

No order effects were observed in analyses of the AIMS data. As Figure 5 shows, the post-phenylalanine increase in TD symptomatology was comparable regardless of whether the active drink was given first or second. The fact that plasma phenylalanine increased to a greater degree on day 2 than day 1 while the magnitude of behavioral change in TD symptoms remained relatively constant, suggests that TD symptoms proved insensitive to increments in plasma phenylalanine above a threshold value. On the other hand, a Spearman rank order correlation revealed a moderate association between the post-challenge increment in plasma phenylalanine and the post-load worsening of TD symptoms ( $\mathbf{r}=$ $0.63, p=.0001$ ), suggesting a moderate degree of association across the two continua.

When evaluating the change over time in the other amino acids, tyrosine and tryptophan, there were only modest, nonsignificant, negative correlations with the post-phenylalanine worsening of TD symptoms (tyrosine: $-0.37, p=0.14$; tryptophan: $-0.33, p=.20$ ).

\section{Memory}

The analysis using memory recall score as the dependent variable revealed that phenylalanine intake did not 
produce any significant change in memory performance, nor did the results show order effects. Collapsing across day of ingestion, the post-placebo recall score averaged $7.9 \pm 3.0$, whereas the post-phenylalanine recall score averaged $7.7 \pm 3.2$.

\section{DISCUSSION}

The aim of this study was to investigate the possibility that dietary intake of phenylalanine could adversely affect TD symptomatology in patients with schizophrenia. A prior study (Gardos et al. 1992) examining the effects of phenylalanine ingestion on TD symptoms in affectively ill patients yielded equivocal evidence of symptom exacerbation. The present study was the first to implement a double-blind, placebo-controlled design and to study effects on schizophrenic patients without affective symptoms. The major research question was whether a $100 \mathrm{mg} / \mathrm{kg}$ loading dose of phenylalanine would acutely worsen the behavioral manifestations of tardive dyskinesia including deficits in memory recall, which are a frequently reported cognitive accompaniment of tardive dyskinesia.

The results strongly supported the study hypothesis that ingestion of phenylalanine would worsen the dyskinetic movements associated with TD. The hypothesis that memory would be adversely affected was not supported. The phenylalanine challenge significantly, consistently, and noticeably worsened the expression of tardive dyskinesia symptomatology, generating an acute increase in TD severity over a 90-minute period. In addition, the increase in TD severity was significantly correlated with the rise in plasma phenylalanine level $(\mathrm{r}=$ 0.63 ), consistent with the possibility of an underlying pathophysiology. The finding of a positive association between plasma phenylalanine and AIMS scores is consistent with results reported by other investigators (Gardos et al. 1992; Richardson et al. 1986, 1989).

It is necessary to consider possible explanations for why phenylalanine loading clearly exacerbated TD symptoms in the present study of schizophrenic patients, in comparison with the more equivocal results obtained in Gardos et al.'s (1992) study of unipolar depressed patients. It is unlikely that the disparity resulted from induction of higher plasma phenylalanine levels in the current sample, since the magnitude of the observed plasma phenylalanine change after the phenylalanine load was similar to the 10 -fold increase that Gardos et al. (1992) observed in unipolar depressive patients. The present findings regarding other LNAAs were identical to those reported by Gardos and colleagues (1992). For example, the finding that a phenylalanine load produced more than a doubling of tyrosine levels was comparable and probably reflects increased conversion of phenylalanine to tyrosine. The decrease in tryptophan levels observed after phenylalanine loading was also comparable across studies.

Several differences in experimental design and sampling procedures may explain why an adverse effect of phenylalanine on TD symptoms emerged more clearly in this study than in Gardos et al.'s (1992). First, the availability of four repeated AIMS assessments including those during a placebo control enhanced measurement precision and statistical power. Second, all AIMS ratings were made 90 minutes post-drink, at a time when our pilot data and other findings (Stegink et al. 1987; Richardson et al. 1989) indicated that peak phenylalanine plasma levels would occur. There were also important differences between the two study samples. First, the current patients were diagnostically homogenous and had clearly established tardive dyskinesia. Second, no females were included in the sample to eliminate differences due to the fact that women have slightly lower plasma phenylalanine levels than men (Koh and Cha 1983). Third, obese patients were also excluded, because it has been reported that obese individuals show higher plasma phenylalanine levels than nonobese controls due to insulin resistance (Caballero and Wurtman 1987). Fourth, schizophrenic patients without affective symptoms composed the sample, in contrast to the mood-disordered patients studied by Gardos et al. (1992).

In the absence of an affectively ill control group that followed the same protocol, it cannot be determined whether the present findings are specific to schizophrenics who have tardive dyskinesia, or whether they would generalize to other psychiatric and neurologic populations. Abnormalities of amino acid metabolism have been reported in various psychiatric and neurologic disorders. Phenylalanine, specifically, has been found to be elevated in Parkinson's disease, Huntington's chorea, hereditary tremor, musculorum deformans, and senile dementia (de Belleroche et al. 1984; Lakke and Teelken 1976), all of which are related to degeneration of dopaminergic neurons or deficiencies involving dopaminergic action in the striatum.

It is also important to consider possible mechanism by which intake of phenylalanine could exacerbate dyskinetic movements. As proposed earlier, intake of phenylalanine in the absence of competing LNAAs can enhance brain phenylalanine influx, degradation to tyrosine and dopamine synthesis and release, stimulating striatal dopamine receptors rendered supersensitive by chronic blockade due to neuroleptics (Gerlach et al. 1974).

Another mechanism by which phenylalanine might alter brain biochemistry, affecting neurotransmission and behavior, is via one of its derivatives, phenylethylamine. As described by Harper (1984), release of phenylethylamine from the degradation of phenylalanine is normally minor. However, when the system is challenged with large amounts of phenylalanine, production of phenylethylamine increases because the body is 
unable to metabolically manage the excess phenylalanine through its usual hydroxylation to tyrosine (Oates et al. 1963). Phenylethylamine's structure and behavioral sequelae have been shown to be similar to those of the sympathomimetic amines, amphetamine, norepinephrine, and dopamine. For example, phenylethylamine has been shown to increase locomotor activity and induce stereotypy (Antelman et al. 1977). Further, after unilateral lesions in the nigrostriatum, the action of phenylethylamine in the nigrostriatal dopaminergic pathways is to induce amphetamine-like ipsilateral rotation (Antelman et al. 1977). These findings suggest that, in addition to having presynaptic effects on dopamine release, phenylethylamine stimulates striatal dopamine receptors directly. Consistent with phenylethylamine's possible involvement in phenylalanineinduced TD exacerbation, Richardson et al. (1989) found that post-loading levels of phenylalanine and phenylethylamine were significantly correlated, and post-load phenylethylamine levels also tended to be correlated with TD severity.

Rather than enhancing dopaminergic activity, it is also possible that phenylalanine loading could affect neurotransmission and motor behavior by inhibiting the synthesis and release of dopamine and serotonin. Two such pathways can be postulated. First, phenylalanine is known to competitively limit the brain influx of tyrosine and tryptophan (serotonin's precursor), and consequently, catecholamine and indoleamine synthesis, by virtue of its greater affinity for their common blood-brain transport system (Pardridge and Choi 1986). Dopamine turnover may also be adversely affected (Bjerkenstedt et al. 1985). PKU constitutes an example in which excess phenylalanine (resulting from insufficient degradation) successfully competes with tyrosine and tryptophan for brain uptake, decreases brain levels of the monoamines, and results in mental retardation (Harper 1984; Pratt 1982).

Very high doses of phenylalanine might also be expected to decrease synthesis and release of dopamine and serotonin via a second pathway. Tyrosine hydroxylase and tryptophan hydroxylase, the rate-limiting enzymes involved in the synthesis of dopamine and serotonin, respectively, can both be competitively inhibited by phenylalanine (Ikeda et al. 1967; Curtius et al. 1981; Katz et al. 1976). Phenylalanine might be especially likely to inhibit tyrosine hydroxylase and interfere with catecholamine synthesis in humans, who hydroxylate phenylalanine very slowly (Maher 1990).

Some evidence even suggests that phenylalanine can have opposite effects on dopaminergic neurotransmission at different doses. Findings in rodents indicate that a low dose of phenylalanine supports dopamine synthesis through its conversion to tyrosine via phenylalanine hydroxylase. A slightly higher dose, in contrast, diminishes dopamine synthesis and release by competitively inhibiting tyrosine hydroxylase (During et al. 1988; Maher 1990). These findings make apparent the need to exercise caution about generalizing the present findings to different dosages of phenylalanine until adequate doseresponse studies have been conducted.

In summary, this research suggests the potential for the amino acid phenylalanine to be involved in the exacerbation, and possibly the development, of tardive dyskinesia. Possible mechanisms for this involvement include direct and indirect effects of phenylalanine loading on the balance and action of neurotransmitters believed to be involved in the pathogenesis of tardive dyskinesia. This research suggests that dietary intake of phenylalanine, unbalanced by other amino acids, can exacerbate tardive dyskinesia symptoms and suggests that further dose-response studies of phenylalanine and other dietary constituents might prove beneficial in the management of TD. Additional research is needed to determine the reliability of these findings and their specificity to TD in schizophrenia.

\section{ACKNOWLEDGMENTS}

This research was supported in part by a VA Merit Review grant to Dr. Bonnie Spring, by The National Institute of Mental Health Grants MH31593, MH40856, MHCRC 43271 to Dr. Nancy C. Andreasen at the Mental Health Clinical Research Center, and a NARSAD Young Investigator Award to Dr. Keith Rogers. I would like to thank Dr. Andreasen for generously providing use of her clinical research center, - both the patients and the facilities. In addition, I would like to thank Dr. Lori Moss, from the North Chicago VA, and Mr. Frank Fleming, BSN, from the University of Iowa Mental Health Clinical Research Center (MH-CRC), for their help in subject recruitment and TD reliability ratings. I would also like to thank Dr. Stephan Arndt for statistical advice.

\section{REFERENCES}

American Psychiatric Association (1987): Diagnostic and Statistical Manual of Mental Disorders (3rd ed. revised). Washington, DC

Antelman SM, Edwards DJ, Lin M (1977): Phenylethylamine: Evidence for a direct, postsynaptic dopamine-receptor stimulating action. Brain Res 127:317-322

Barnes TRE (1987): The present state of tardive dyskinesia and akathisia in the treatment of schizophrenia. Psychiatric Dev 4:301-319

Benson DF, Blumer D (1982): Psychiatric Aspects of Neurologic Disease, vol 2. New York, Grune \& Stratton

Bjerkenstedt L (1990): Decreased tyrosine transport in schizophrenic patients. In Richardson MA (ed), Amino Acids in Psychiatric Disease. Washington, DC, American Psychiatric Press, pp 161-170

Bjerkenstedt L, Edman G, Hagenfeldt L, Sedvall G, Wiesel FA (1985): Plasma amino acids in relation to cerebrospinal fluid monoamine metabolites in schizophrenic patients and healthy controls. Br J Psychiatry 147:276-282 
Caballero B, Wurtman RJ (1987): Control of plasma phenylalanine levels. In Wurtman RJ, Ritter-Walker E (eds), Dietary Phenylalanine and Brain Function. Boston, Birkhauser, pp 9-23

Carroll BJ, Curtis GC, Keekmen E (1977): Paradoxical response to dopamine agonists in tardive dyskinesia. Am J Psychiatry 1134:785-789

Casey DE (1987): Tardive dyskinesia. In Meltzer HY (ed), Psychopharmacology: The Third Generation of Progress. New York, Raven Press, pp 1411-1419

Curtius $\mathrm{H}-\mathrm{CH}$, Niederwieser A, Viscontini M, Leimbacher W, Wegman H, Blehova B, Rey F, Schaub J, Schmidt H (1981): Serotonin and dopamine synthesis in phenylketonuria. Adv Exp Med Biol 133:277-291

de Belleroche J, Recordati A, Clifford Rose, F (1984): Elevated levels of amino acids in the CSF of motor neuron disease patients. Neurochem Pathol 2:1-6

DeVeaugh-Geiss J (1982): Tardive Dyskinesia and Related Involuntary Movement Disorders. Boston, John Wright

During MJ, Acworth IN, Wurtman RJ (1988): Phenylalanine administration influences dopamine release in the rat's corpus striatum. Neurosci Lett 93:91-95

Ebadi M, Hama Y (1988): Dopamine, GABA, cholecystokinin and opioids in neuroleptic-induced tardive dyskinesia. Neurosci Biobehav Rev 12:179-187

Fernstrom JD, Faller DV (1978): Neutral amino acids in the brain: Changes in response to food ingestion. J Neurochemistry 30:1531-1538

Fernstrom JD, Wurtman RJ (1972): Brain serotonin content: Physiological regulation by plasma neutral amino acids. Science 178:414-416

Fibiger HC, Lloyd KG (1984): Neurobiological substrates of tardive dyskinesia: The GABA hypothesis. Trends Neurosci 7:462-464

Gardos G, Cole JO, Matthews JD, Nierenberg AA, Dugan SJ (1992): The acute effects of a loading dose of phenylalanine in unipolar depressed patients with and without tardive dyskinesia. Neuropsychopharmacology 6:241-247

Gerlach J, Reisby N, Randrup A (1974): Dopaminergic hypersensitivity and cholinergic hypofunction in the pathophysiology of tardive dyskinesia. Psychopharmacologia $34: 21-35$

Hagenfeldt L, Bjerkenstedt L, Edman G, Sedvall G, Wiesel F-A (1984): Amino acids in plasma and CSF and monoamine metabolites in CSF: Interrelationship in healthy subjects. J Neurochemistry 42:833-837

Harper AE (1984): Phenylalanine metabolism. In Stegink LD, Filer LJ Jr (eds), Aspartame: Physiology and Biochemistry. New York, Marcel Dekker, pp 77-109

Hernandez T, Coulson RA (1961): The effect of insulin on amino acid metabolism. Biochemistry J 79:596

Ikeda M, Levitt M, Udenfriend S (1967): Phenylalanine as a substrate and inhibitor of tyrosine hydroxylase. Arch Biochem Biophys 120:420-427

Jeste DV, Wyatt RJ (1981): Changing epidemiology of tardive dyskinesia: An overview. Am J Psychiatry 138:297-309

Kane JM, Smith JM (1982): Tardive dyskinesia: Prevalence and risk factors, 1959-1979. Arch Gen Psychiatry 39:473-481
Kane JM, Woerner M, Lieberman JA, Weinhold P, Florio W, Rubinstein M, Rotrosen J, Kurucz J, Mukherjee S, Bergman. K, Schooler NR (1985): The prevalence of tardive dyskinesia. Psychopharmocol Bull 21:136-139

Katz I, Lloyd T, Kaufman S (1976): Studies on phenylalanine and tyrosine hydroxylation by rat brain tyrosine hydroxylase. Biochim Biophys Acta 445:567-578

Koh ET, Cha CJ (1983): Comparison of plasma amino acids by race, sex, and age. Nutr Rep Int 28:8-22

Krause W, Halminski M, McDonald L, Dembure P, Salvo R, Freides D, Elsas L (1985): Biochemical and neuropsychological effects of elevated plasma phenylalanine in patients with treated phenylketonuria. J Clin Invest 75:40-48

Lakke JPWF, Teelken AW (1976): Amino acid abnormalities in cerebrospinal fluid of patients with Parkinsonism and extrapyramidal disorders. Neurology 26:489-493

Lenda K, Svenneby G (1980): Rapid high-performance liquid chromatographic determination of amino acids in synaptosomal extracts. J Chromatogr 198:516-519

Lindroth P, Mopper K (1979): High performance liquid chromatographic determination of subpicomole amounts of amino acids by precolumn fluorescence derivatization with ophthalidialdehyde. Anal Chem 51:1667-1674

Lippman RW (1958): The significance of heterozygocity for hereditary metabolic errors related to mental deficiency (oliqomentia). Am J Ment Defic 63:320-324

Maher TJ (1990): Effects of phenylalanine on the synthesis, release, and function of catecholaminergic systems. In Richardson MA (ed), Amino Acids in Psychiatric Disease. Washington, DC, American Psychiatric Press

Melamed E, Hefti F, Wurtman RJ (1980): Tyrosine administration increases striatal dopamine release in rats with partial nigrostriatal lesions. Proc Nat Acad Sci 77:4305-4309

Milner JD, Irie K, Wurtman RJ (1986): Effects of phenylalanine on the release of endogenous dopamine from rat striatal slices. J Neurochemistry 47:1444-1448

Myslobodsky MS, Tomer R, Holden T, Kempler S, Sigal M (1985): Cognitive impairment in patients with tardive dyskinesia. J Nerv Ment Dis 173:156-160

Nasrallah HA, Dunner FJ, McCalley-Whitters M, Smith RE (1986): Pharmacologic probes of neurotransmitter systems in tardive dyskinesia: Implications for clinical management. J Clin Psychiatry 47:56-59

National Institute of Mental Health, Psychopharmacology Research Branch (1975): Development of a dyskinetic movement scale. ECDEU Intercom 4:(1)3-6

Oates JA, Nirenberg PZ, Jepson JB, Sjoerdsma A, Udenfriend $S$ (1963): Conversion of phenylalanine to phenylethylamine in patients with PKU. Proc Soc Exp Biol Med 112:1078-1081

Oldfield, RC (1971): The assessment and analysis of handedness: The Edinburgh Inventory. Neuropsychologia 9:97-113

Owens DGC, Johnstone EC, Frith CD (1982): Spontaneous involuntary disorders of movement: Their prevalence, severity, and distribution in chronic schizophrenics with and without treatment with neuroleptics. Arch Gen Psychiatry 39:452-461 
Pardridge WM, Choi TB (1986): Neutral amino acid transport at the human blood-brain barrier. Fed Proc 45:2073-2078

Pardridge WM, Oldendorf WH (1977): Transport of metabolic substrates through the blood-brain barrier. J Neurochem 28:5-12

Poisner AM (1960): Serum phenylalanine in schizophrenia: Biochemical genetic aspects. J Nerv Ment Dis 131:74-76

Potkin SG, Cannon-Spoor HE, DeLisi LE, Neckers LM, Wyatt RJ (1983): Plasma phenylalanine, tyrosine, and tryptophan in schizophrenia. Arch Gen Psychiatry 40:749-752

Pratt OE (1982): Transport inhibition in the pathogenesis of phenylketonuria and other inherited metabolic diseases. J Inherited Metab Dis 2(Suppl):75-81

Reveley MA, De Belleroche J, Recordati A, Hirsch SR (1987): Increased CSF amino acids and ventricular enlargement in schizophrenia: A preliminary study. Biol Psychiatry 22:413-420

Rey A (1964): L'examen clinique en psychologie. Paris, Presses Universitaires de France

Richardson MA, Casey DE (1988): Tardive dyskinesia status: Stability or change. Psychopharmocol Bull 24:471-475

Richardson MA, Haugland G, Pass R, Craig TJ (1986): The prevalence of tardive dyskinesia in a mentally retarded population. Psychopharmacol Bull 22:243-249

Richardson MA, Suckow R, Whittaker R, Boggiano W, Sziraki I, Kushner H, Perumal A (1989): The plasma phenylalanine/large neutral amino acid ratio: A risk factor for tardive dyskinesia. Psychopharmacol Bull 25:1-12

Saltz BL, Kane JM, Woerner MG, Lieberman JA, Alvir J, Blank K, Kahaner K, Foley C (1989): Prospective study of tardive dyskinesia in the elderly. Psychopharmacol Bull 25:52-56

Schooler N, Kane J (1982): Research diagnosis for tardive dyskinesia (RD-TD). Arch Gen Psychiatry 39:486-487

Simpson GM, Lee JH, Zoubok B, Gardos G (1979): A rating scale for tardive dyskinesia. Psychopharmacology 64:171-179

Smith JM, Baldessarini RJ (1980): Changes in prevalence, severity, and recovery in tardive dyskinesia with age. Arch Gen Psychiatry 37:1368-1373

Serokin JE, Giordani B, Mohs RC, Losonczy MF, Davidson M, Siever LJ, Ryan TA, Davis KL (1988): Memory impairment in schizophrenic patients with tardive dyskinesia. Biol Psychiatry 23:129-135

Stegink LD, Filer LJ (1984): Aspartame: Physiology and Biochemistry. New York, Marcel Dekker

Stegink LD, Wolf-Novak LC, Filer LJ, Bell EF, Ziegler EE, Kraus WL, Brummel MC (1987): Aspartame-sweetened beverage: Effect on plasma amino acid concentrations in normal adults. Journal of Nutrition 117:1989-1995

Taylor EM (1959): The Appraisal of Children with Cerebral Deficits. Cambridge, MA, Ballinger

Wade JB, Taylor MA, Kasprisin A, Rosenberg S, Fiducia D (1987): Tardive dyskinesia and cognitive impairment. Biol Psychiatry 22:393-395

Wolf ME, Mosnaim AD (1988): Tardive Dyskinesia: Biological Mechanisms and Clinical Aspects. Washington, DC, American Psychiatric Press

Wurtman RJ (1983): Neurochemical changes following highdose aspartame with dietary carbohydrates. N Engl J Med 309:429-430

Yassa R, Nair NPV, Iskandar H, Schwartz G (1990): Factors in the development of severe forms of tardive dyskinesia. Am J Psychiatry 147:1156-1163 